\title{
Mathematical Eco-Epidemiological Model on Prey-Predator System
}

\author{
Abayneh Fentie Bezabih ", Geremew Kenassa Edessa, Purnachandra Rao Koya \\ Department of Mathematics, Wollega University, Nekemte, Ethiopia \\ Email address: \\ abaynehf@hu.edu.et (A. F. Bezabih), gbonsa.kena@gmail.com (G. K. Edessa), drkpraophd@gmail.com (P. R. Koya) \\ ${ }^{*}$ Corresponding author
}

\section{To cite this article:}

Abayneh Fentie Bezabih, Geremew Kenassa Edessa, Purnachandra Rao Koya. Mathematical Eco-Epidemiological Model on Prey-Predator System. Mathematical Modelling and Applications. Vol. 5, No. 3, 2020, pp. 183-190. doi: 10.11648/j.mma.20200503.17

Received: June 2, 2020; Accepted: June 23, 2020; Published: August 20, 2020

\begin{abstract}
This paper presents infectious disease in prey-predator system. In the present work, a three Compartment mathematical eco-epidemiology model consisting of susceptible prey- infected prey and predator are formulated and analyzed. The positivity, boundedness, and existence of the solution of the model are proved. Equilibrium points of the models are identified. Local stability analysis of Trivial, Axial, Predator-free, and Disease-free Equilibrium points are done with the concept of Jacobian matrix and Routh Hourwith Criterion. Global Stability analysis of endemic equilibrium point of the model has been proved by defining appropriate Liapunove function. The basic reproduction number in this eco-epidemiological model obtained to be $R_{o}=\left[\beta\left(\mu_{3}\right)^{2}\right] /\left[q p_{2}\left(q p_{1} \Lambda-\mu_{1} \mu_{3}\right)\right]$. If the basic reproduction number $R_{o}>1$, then the disease is endemic and will persist in the prey species. If the basic reproduction number $R_{o}=1$, then the disease is stable, and if basic reproduction number $R_{o}<1$, then the disease is dies out from the prey species. Lastly, Numerical simulations are presented with the help of DEDiscover software to clarify analytical results.
\end{abstract}

Keywords: Mathematical Ecoepidemiology, Prey- Predator System, Stability Analysis, Reproduction Number, Simulation Study

\section{Introduction}

Mathematical Ecology and Mathematical epidemiology are two major fields in the study of biology and applied mathematics. However, as time goes these two fields are more and more closer, and a new cross field which is called Mathematical eco-epidemiology emerges [1-7]. In recent years, Mathematical eco-epidemiology problem has got more attention to many scholars and experts $[2,7]$.

Anderson R and May R. [6, 7, 10] were the first to propose an eco-epidemiological model by merging the Lotka-volterra prey-predator model $[2,7,10]$ and the epidemiological SIR model which was introduced by Kermack and Mckendrick. [2, 7, 12-14]. Many works have been devoted to study infectious disease on a prey-predator system [1-10]. Most models for the transmission of infectious diseases originated from the classic work of Kermack-McKendrick. In the last few decades, mathematical models have become extremely important tools in understanding and analyzing the spread and control of infectious disease $[8,9]$.
Ecological populations suffer from various diseases. These diseases often play an important roles in regulating the population sizes $[1,5,10,12,13]$. Mathematical study of such populations has attracted attentions of both ecologists and mathematicians from several years past. As a result numerous mathematical models have been developed, and these models have become essential tools in analyzing the interaction of different populations, particularly the interaction between prey and predator $[10,12,15,17]$.

For predator-prey ecosystems, infectious diseases coupled with predator-prey interaction to produce a complex combined effect as regulators of predator and prey population sizes $[1-5,10,12,18]$. Infectious Disease transmission and interaction of species in a certain ecological environment can't be neglected, it is very crucial to observe the dynamics of ecological interaction of species with infectious disease. Disease may spread among prey and predator species, so it vital to study a prey-predator system in particular with infection in prey population.

In this study, the effect of disease in ecological system is 
an important issue from a mathematical as well as an ecological point of view. So A Mathematical ecoepidemiological model is constructed by Considering well known model Lotka-Volterra Predator-Prey model from mathematical ecology and Kermack-McKendrick model from mathematical epidemiology. More detailed assumptions and descriptions can be seen in the next section.

This paper is organized as follows. In Section 2, an ecoepidemiological model with disease in prey species are formulated and some primarily results are discussed (positivity, boundedness, and existence of solution of the model). In Section 3, Main results and Discussion: all possible equilibrium points of the model is investigated. Local stability of equilibrium points are done using Next generation matrix and Routh Hourwith criterion. Global stability analysis is performed by taking appropriate liapunove function. In Section 4, Numerical simulations are done to clarify theoretical results. Finally brief conclusions are given in Section 5 .

\section{Model Formulation and Assumptions}

Let $X(t)$ be the total population density of the prey species and $P(t)$ be the population density of the predator species. Now, to formulate mathematical eco-epidemiological model, the following assumptions are drawn:

i. The total prey $X(t)$ is composed of two classes: susceptible prey $(S)$ and infective prey $(I)$, the total population of the prey species $X(t)=S(t)+I(t)$.

ii. The disease spreads among the prey population only by contact, and can't be transmitted vertically. The infected prey species do not recover or become immune. The incidence of the disease in the prey is bilinear incidence form $\beta S I$.

iii. The predation functional response of the predator towards susceptible prey as well as infected prey are assumed to follow Simple bilinear functional form with $\mathrm{p}_{1}, \mathrm{p}_{2}$, be respective predation coefficients and Consumed prey is converted into predator with efficiency $q$.

iv. The susceptible prey and the infected prey are both captured by predators. susceptible prey are more stronger than infected prey. Therefore, the probability of predation of the infected prey is more than that of susceptible prey. That is $p_{2}>p_{1}$.

v. Suppose that susceptible prey, infected prey and predator have different mortality rates.

$\mu_{1}, \mu_{2}, \mu_{3}$ respectively. Furthermore infected prey Suffer from death rate due to infection $\delta$.

vi. All parameters are assumed to be positive constants.

Table 1. Notation and Description of Model Variables.

\begin{tabular}{ll}
\hline Variables & Descriptions \\
\hline $\mathrm{S}(\mathrm{t})$ & Population size ofthe susceptible preyat timet \\
$\mathrm{I}(\mathrm{t})$ & Population size ofthe infective prey at time $t$ \\
$\mathrm{P}(\mathrm{t})$ & Population size of the predator at time $t$ \\
\hline
\end{tabular}

Table 2. Notations and Description of model parameters.

\begin{tabular}{ll}
\hline Parameter & Description of parameter \\
\hline$\Lambda$ & The constant recruitment rate of susceptible prey \\
$\beta$ & The disease transmission coefficient \\
$\mathrm{p}_{1}$ & Capture rate of the susceptible prey \\
$\mathrm{p}_{2}$ & Capture rate of the infected prey \\
$q$ & Conversion coefficient from the prey to the predator \\
$\mu_{1}$ & The death rate of the susceptible prey \\
$\mu_{2}$ & The death rate of the infected prey \\
$\mu_{3}$ & The death rate of the predator \\
\hline
\end{tabular}

According to the above assumptions, the description of variables and parameters the present model will have the flow diagram given in Figure 1.

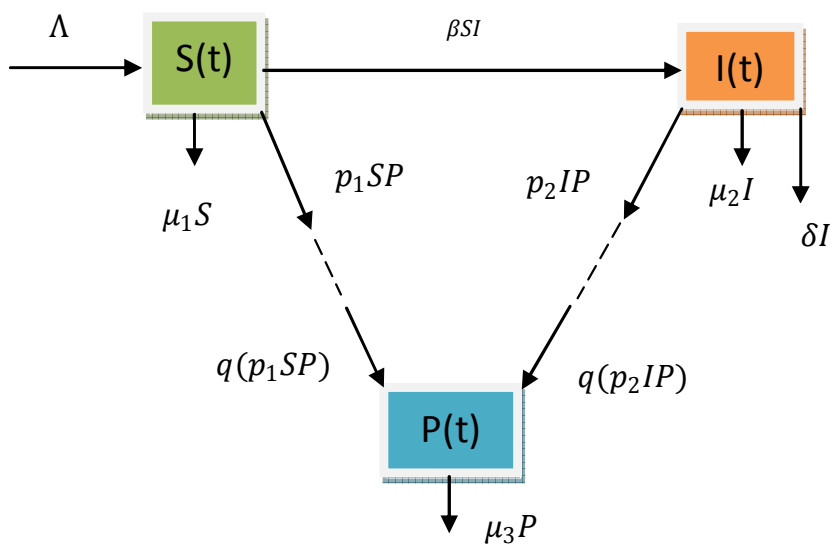

Figure 1. Model Diagram.

The model flow diagram shown in Figure 1 leads to the following system of three ordinary differential equations.

$$
\begin{aligned}
& d S / d t=\Lambda-\beta S I-p_{1} S P-\mu_{1} S \\
& d I / d t=\beta S I-p_{2} I P-\mu_{2} I-\delta I \\
& d P / d t=q p_{1} S P+q p_{2} I P-\mu_{3} P
\end{aligned}
$$

with initial conditions $s(0) \geq 0, I(0) \geq 0, P(0) \geq 0$

In this section, it is shown the basic properties which are necessary for understanding of subsequent results. So the following lemmas are stated as primarily results.

Lemma 1 [Positivity] All solution of the model (1)-(3) with initial conditions are positive for all time $t$

Proof: Positivity of the model variables is shown separately for each of the model variables $S(t), I(t)$, and $R(t)$.

Positivity of $S(t)$ :

The model equation (1) given by $d S / d t=\Lambda-\beta S I-$ $p_{1} S P-\mu_{1} S$ can be expressed without loss of generality, after eliminating the positive terms $(\Lambda)$ which are appearing on the right hand side, as an inequality as $d S / d t \geq$ $-\left(\beta I+p_{1} P+\mu_{1}\right) S$. Using variables separable method and on applying integration, the solution of the foregoing differentially inequality can be obtained as $S(t) \geq$ $e^{-\left(\beta I+p_{1} P+\mu_{1}\right) t}$. Recall that an exponential function is always non-negative irrespective of the sign of the exponent, Hence, it can be concluded that $S(t) \geq 0$.

Positivity of $I(t)$ : 
The model equation (2) arranged $d I / d t=\beta S I-p_{2} I P-$ $\mu_{2} I-\delta I$ can be expressed without loss of generality, after eliminating the positive term $(\beta S I)$ which are appearing on the right hand side, as an inequality as $d I / d t \geq-\left(p_{2} P+\right.$ $\left.\mu_{2}+\delta\right) I$. Using variables separable method and on applying integration, the solution of the foregoing differentially inequality can be obtained as $I(t) \geq e^{-\left(p_{2} P+\mu_{2}+\delta\right) t}$. Recall that an exponential function is always non-negative irrespective of the sign of the exponent. Hence, it can be concluded that $I(t) \geq 0$.

Positivity of $P(t)$ :

The model equation (3) arranged $d P / d t=q p_{1} S P+$ $q p_{2} I P-\mu_{3} P$ can be expressed without loss of generality, after eliminating the positive term $\left(q p_{1} S P+q p_{2} I P\right)$ which are appearing on the right hand side, as an inequality as $d P / d t \geq-\left(\mu_{3}\right) P$. Using variables separable method and on applying integration, the solution of the foregoing differentially inequality can be obtained as $P(t) \geq e^{-\left(\mu_{3}\right) t}$. Recall that an exponential function is always non-negative irrespective of the sign of the exponent. Hence, it can be concluded that $P(t) \geq 0$.

Lemma 2 [Boundedness] All feasible regions $\Omega$ defined by $\Omega=\left\{(S(t), \quad I(t), \quad P(t)) \in \mathbb{R}_{+}^{3}: S(t)+I(t)+P(t) \leq\right.$ $\Lambda / \eta=N\} \quad$ where $\eta=\min \left\{\begin{array}{lll}\mu_{1} & \mu_{2} & \mu_{3}\end{array}\right\}$ with initial conditions $S(t) \geq 0, S(t) \geq 0, S(t) \geq 0$ is positively invariant for the model (1)-(3)

Proof: Define a function $N(t)=S(t)+I(t)+P(t)$, we have that $d N / d t=[d S / d t]+[d I / d t]+[d P / d t]$

$$
\begin{gathered}
\frac{d N}{d t}=\left[\Lambda-\beta S I-p_{1} S P-\mu_{1} S\right]+\left[\beta S I-p_{2} I P-\mu_{2} I\right] \\
+\left[q p_{1} S P+q p_{2} I P-\mu_{3} P\right] \leq \Lambda+q p_{1} S P+q p_{2} I P \\
{[d N / d t]+\eta N=\Lambda+\left(\eta-p_{1} P-\mu_{1}\right) S+\left(\eta-p_{2} I-\mu_{2}\right) I} \\
+\left[\eta-\mu_{3}+\left(p_{1} S+p_{2} I\right) q\right] P
\end{gathered}
$$

$[d N / d t]+\eta N \leq \Lambda$ The solution ofsuch ode is given by $N(t) \leq[\Lambda / \eta]+C \exp (-\eta t)$ limit of $\mathrm{N}(\mathrm{t})$ as $\mathrm{t}$ tends to $+\infty \quad$ is $\quad \Lambda / \eta$ hence $0 \leq N(t) \leq \Lambda / \eta$. Then $\Omega=\left\{(S(t), \quad I(t), \quad P(t)) \in \mathbb{R}_{+}^{3}: S(t)+I(t)+P(t) \leq\right.$ $\Lambda / \eta=N\}$ is a positive invariant set of system.

Lemma 3 [existence of the solutions] Solutions of the model equations (1) - (3) together with the initial conditions $S(0)>0, I(0) \geq 0, P(0) \geq 0$ exist in $\mathbb{R}_{+}^{3}$ i.e., the model variables $S(t), I(t)$ and $P(t)$ exist for all $t$ and will remain in $\mathbb{R}_{+}^{3}$.

Proof: Let the system of equation (1) - (3) arranged as follows:

$$
\begin{gathered}
f_{1}=\Lambda-\beta S I-p_{1} S P-\mu_{1} S \\
f_{2}=\beta S I-p_{2} I P-\mu_{2} I-\delta I \\
f_{3}=q p_{1} S P+q p_{2} I P-\mu_{3}
\end{gathered}
$$

According to Derrick and Grossman theorem, let $\Omega$ denote the region $\Omega=\left\{(S, I, P) \in \mathbb{R}_{+}^{3} ; \mathrm{N} \leq(\Lambda / \mu)\right\}$. Then equations (1) - (3) have a unique solution if $\left(\partial f_{i}\right) /\left(\partial x_{j}\right), i, j=1,2,3$ are continuous and bounded in $\Omega$. Here, $x_{1}=S, x_{2}=I, x_{3}=P$, The continuity and the boundedness are shown as follows:

For $f_{1}$ :

$$
\begin{gathered}
\left|\left(\partial f_{1}\right) /(\partial S)\right|=\left|-\beta \mathrm{I}-\mathrm{p}_{1} \mathrm{P}-\mu_{1}\right|<\infty \\
\left|\left(\partial f_{1}\right) /(\partial I)\right|=|-\beta S|<\infty \\
\left|\left(\partial f_{1}\right) /(\partial P)\right|=\left|-\mathrm{p}_{1} \mathrm{~S}\right|<\infty
\end{gathered}
$$

For $f_{2}$ :

$$
\begin{gathered}
\left|\left(\partial f_{2}\right) /(\partial S)\right|=|\beta \mathrm{I}|<\infty \\
\left|\left(\partial f_{2}\right) /(\partial I)\right|=\left|\beta S-\mathrm{p}_{2} \mathrm{P}-\mu_{2}-\delta\right|<\infty \\
\left|\left(\partial f_{2}\right) /(\partial P)\right|=\left|-\mathrm{p}_{2} \mathrm{I}\right|<\infty
\end{gathered}
$$

For $f_{3}$ :

$$
\begin{gathered}
\left|\left(\partial f_{3}\right) /(\partial S)\right|=\left|\mathrm{qp}_{1} \mathrm{P}\right|<\infty, \\
\left|\left(\partial f_{3}\right) /(\partial I)\right|=\left|\mathrm{qp}_{2} \mathrm{P}\right|<\infty, \\
\left|\left(\partial f_{3}\right) /(\partial P)\right|=\left|\mathrm{qp}_{1} \mathrm{~S}+\mathrm{qp}_{2} \mathrm{I}-\mu_{3}\right|<\infty
\end{gathered}
$$

\section{Main Results and Discussion}

\subsection{Equilibrium Points}

In this section, the existence of equilibrium points and their stability Analysis will be discussed. Model Equations (1) - (3) have the following Equilibrium points: (i) Trivial equilibrium point $E_{0}(0,0,0)$, (ii) Axial equilibrium point $E_{1}(S, 0,0)=\left(\Lambda / \mu_{1}, 0,0\right) \quad, \quad$ (iii) Predator-free /predator extinction equilibrium point.

$$
E_{2}(S, \quad I, \quad 0)=\left(\mu_{2} / \beta, \quad\left[\beta \Lambda-\mu_{1} \mu_{2}\right] /\left[\beta \mu_{2}\right], 0\right),
$$

(iv) Disease-free equilibrium point $E_{3}\left(\begin{array}{lll}\bar{S} & 0 & \bar{p}\end{array}\right)=$ $\left(\mu_{3} / q p_{1}, \quad 0, \quad\left[q p_{1} \Lambda-\mu_{1} \mu_{3}\right] /\left[p_{1} \mu_{3}\right]\right), \quad$ (v) positive /endemic equilibrium point $E_{4}\left(S^{*}, I^{*}, P^{*}\right)$.

\subsection{Basic Reproduction Number}

The Next generation matrix method $[5,18]$ is used to calculate the basic reproduction number $R_{0}$ [5]. it is Clearly, $I$ is the only relevant class of infection. The class $I(t)$ from our model (2) can be expressed as follows.

$$
d I / d t=\beta S I-p_{2} I P-\mu_{2} I-\delta I=\left\{\beta S-\left(p_{2} P+\mu_{2}+\right.\right.
$$

$\delta\} I$. Therefore, two matrices $\mathrm{F}$ and $\mathrm{V}$ corresponding to the gain and loss components of equation (2) Can be defined as $F=\beta S, V=p_{2} P+\mu_{2}+\delta$ The matrices evaluated at disease-free equilibrium point, $E_{3}\left(\begin{array}{lll}\bar{S} & 0 & \bar{P}\end{array}\right), F=\beta \bar{S}, V=$ $p_{2} \bar{P}+\mu_{2}+\delta$ Now, the Next generation matrix is defined as $G=F V^{-1}$. The basic reproduction numberis thedominant eigen value of the next generation matrix. Thus, $R_{o}=$ $(\beta \bar{S}) /\left(p_{2} \bar{P}+\mu_{2}+\delta\right)$, Where $\bar{S}=\mu_{3} / q p_{1}$, and $\bar{P}=$ $\left[q p_{1} \Lambda-\mu_{1} \mu_{3}\right] /\left[p_{1} \mu_{3}\right] \quad$ Then $R_{o}=\left[\beta\left(\mu_{3}\right)^{2}\right] /\left[q p_{2}\left(q p_{1} \Lambda-\mu_{1} \mu_{3}\right)\right]$. If $R_{o}>1$, then the disease is endemic and will persist in the prey species. If $R_{o}=1$ then the disease is stable, and if $R_{o}<1$, then the disease is dies out from the prey species.

Next, to investigate the stability analysis of equilibrium 
points of the model (1)-(3) have to be represented by functions:

$$
\begin{aligned}
& d S / d t=\Lambda-\beta S I-p_{1} S P-\mu_{1} S \equiv F(S, \quad I, \quad P) \\
& d I / d t=\beta S I-p_{2} I P-\mu_{2} I-\delta I \equiv G(S, \quad I, \quad P) \\
& d P / d t=q p_{1} S P+q p_{2} I P-\mu_{3} P \equiv H(S, \quad I, \quad P)
\end{aligned}
$$

The Jacobian matrix of the above system of functions is given by $J(S, \quad I, \quad P)=\left(\begin{array}{lll}F_{S} & F_{I} & F_{P} \\ G_{S} & G_{I} & G_{P} \\ H_{S} & H_{I} & H_{P}\end{array}\right)$

$$
=\left(\begin{array}{ccc}
-\beta \mathrm{I}-\mathrm{p}_{1} \mathrm{P}-\mu_{1} & \mathrm{~J}, \mathrm{I}, \mathrm{P}) & -\mathrm{p}_{1} \mathrm{~S} \\
\beta \mathrm{I} & \beta S-\mathrm{p}_{2} \mathrm{P}-\mu_{2}-\delta & -\mathrm{p}_{2} \mathrm{I} \\
\mathrm{qp}_{1} \mathrm{P} & \mathrm{qp}_{2} \mathrm{P} & \mathrm{qp}_{1} \mathrm{~S}+\mathrm{qp}_{2} \mathrm{I}-\mu_{3}
\end{array}\right)
$$

With help of matrix $J$, it is possible to analyze the local stability of equilibrium points. In this model, it is possible to verify that trivial equilibrium $E_{0}(0,0,0)$ is always stable.

Theorem 1 [Trivial Equilibrium point] $E_{0}(0,0,0)$ is locally asymptotically stable for all parameters.

Proof: Evaluate the Jacobian matrix at $E_{0} J\left(E_{0}\right)=$ $\left(\begin{array}{ccc}-\mu_{1} & 0 & 0 \\ 0 & -\left(\mu_{2}+\delta\right) & 0 \\ 0 & 0 & -\mu_{3}\end{array}\right)$ The Eigen values of the Jacobian matrix $J$ at $E_{0}$ are $-\mu_{1},-\left(\mu_{2}+\delta\right),-\mu_{3}$ which is all eigen values are negative, hence trivial equilibrium point is locally asymptotically stable for all parameters.

Theorem 2 [Axial equilibrium point] $E_{1}(S, 0,0)=$ $\left(\Lambda / \mu_{1}, 0,0\right)$ is locally asymptotically stable if $\mu_{1} \mu_{2} \geq$ $\beta \Lambda$ and $\mu_{1} \mu_{3} \geq q p_{1} \Lambda$, otherwise unstable.

Proof: The Jacobian matrix at axial equilibrium point $E_{1}$ is given by $J(S, \quad 0,0)=\left(\begin{array}{ccc}-\mu_{1} & -\beta S & -p_{1} S \\ 0 & \beta S-\mu_{2} & 0 \\ 0 & 0 & q p_{1} S-\mu_{3}\end{array}\right)$ axial equilibrium point $E_{1}$ is stableif $\beta S-\mu_{2}<0$ and $q p_{1} S-$ $\mu_{3}<0$ i.e. $\beta\left(\Lambda / \mu_{1}\right)-\mu_{2}<0$ and $q p_{1}\left(\Lambda / \mu_{1}\right)-\mu_{3}<$ 0 andhence $\mu_{1} \mu_{2} \geq \beta \Lambda$ and $\mu_{1} \mu_{3} \geq q p_{1} \Lambda$ holds true, otherwise unstable

Theorem 3The predator-extinction /predator-free equilibrium point $E_{2}(S, I, \quad 0)=\left(\mu_{2} / \beta, \quad\left[\beta \Lambda-\mu_{1} \mu_{2}\right] /\left[\beta \mu_{2}\right], 0\right)$ is locally asymptotically stable if the following conditions are satisfied:

i. $q p_{1} S+q p_{2} I-\mu_{3}<0$,

ii. $\beta I+\mu_{1}+\mu_{2}-\beta S>0 \&$

$\left(\beta I+\mu_{1}\right)\left(\mu_{2}-\beta S\right)+\beta^{2} S I>0$ i.e.

$q\left[p_{1} \mu_{2}^{2}+p_{2}\left(\beta \Lambda-\mu_{1} \mu_{2}\right)\right]<\beta \mu_{2} \mu_{3}$,

$\beta\left[\mu_{2}{ }^{2}-\left(\beta \Lambda-\mu_{1} \mu_{2}\right)\right]<\beta \mu_{2}\left(\mu_{1}+\mu_{2}\right)$ and

$\mu_{1} \mu_{2}<\beta \Lambda$ holds true. Otherwise unstable.

Proof: The Jacobian matrix at $E_{2} J(S, I, 0)$

$$
\left(\begin{array}{ccc}
-\beta I-\mu_{1} & -\beta S & -p_{1} S \\
\beta I & \beta S-\mu_{2} & -p_{2} I \\
0 & 0 & q p_{1} S+q p_{2} I-\mu_{3}
\end{array}\right)
$$

To find Eigen value of the Jacobian matrix

$$
\operatorname{Det}\left[J\left(E_{2}\right)-\lambda I\right]
$$

$$
\left|\begin{array}{ccc}
-\beta I-\mu_{1}-\lambda & -\beta S & -p_{1} S \\
\beta I & \beta S-\mu_{2}-\lambda & -p_{2} I \\
0 & 0 & q p_{1} S+q p_{2} I-\mu_{3}-\lambda
\end{array}\right|
$$

which results a polynomial. $\left(q p_{1} S+q p_{2} I-\mu_{3}-\lambda\right)\left[\left(-\beta I-\mu_{1}-\lambda\right)\left(\beta S-\mu_{2}-\lambda\right)+\right.$ $\left.\beta^{2} \mathrm{SI}\right]=0$ and can be written as

$$
\begin{gathered}
\left(q p_{1} S+q p_{2} I-\mu_{3}-\lambda\right) \\
{\left[\left(\lambda+\beta I+\mu_{1}\right)\left(\lambda+\mu_{2}-\beta S\right)+\beta^{2} S I\right]=0 .}
\end{gathered}
$$

Now, the system is said to be stable if all Eigen values of the polynomial is negative, otherwise unstable. hence $q p_{1} S+q p_{2} I-\mu_{3}<0$ and the remaining Eigen values is the roots of quadratic equation

$\left(\lambda+\beta I+\mu_{1}\right)\left(\lambda+\mu_{2}-\beta S\right)+\beta^{2} S I=0$, Routh Hourwith criterion results

$$
\begin{gathered}
\beta I+\mu_{1}+\mu_{2}-\beta S>0 \&\left(\beta I+\mu_{1}\right)\left(\mu_{2}-\beta S\right)+\beta^{2} \text { SI }>0, \\
q\left[p_{1} \mu_{2}^{2}+p_{2}\left(\beta \Lambda-\mu_{1} \mu_{2}\right)\right]<\beta \mu_{2} \mu_{3}
\end{gathered}
$$

$\beta\left[\mu_{2}{ }^{2}-\left(\beta \Lambda-\mu_{1} \mu_{2}\right)\right]<\beta \mu_{2}\left(\mu_{1}+\mu_{2}\right)$ and $\mu_{1} \mu_{2}<\beta \Lambda$, holds true, Otherwise unstable.

Theorem 4. [The Disease-free equilibrium point]

$E_{3}(S, 0, P)=\left(\mu_{3} / q p_{1}, \quad 0, \quad\left[q p_{1} \Lambda-\mu_{1} \mu_{3}\right] /\left[p_{1} \mu_{3}\right]\right)$ is globally asymptotically stable if the following conditions are satisfied:

i. $\beta S-p_{2} P-\mu_{2}<0$,

ii. $p_{1} P+\mu_{1}+\mu_{3}-q p_{1} S>0$, and $\left(p_{1} P+\mu_{1}\right)\left(\mu_{3}-q p_{1} S\right)+\mathrm{qp}_{1}{ }^{2} \mathrm{SP}>0$ i.e.

$\beta \mu_{3}-q p_{2}\left(q p_{1} \Lambda-\mu_{1} \mu_{3}\right)<q p_{1} \mu_{2} \mu_{3}$, $q p_{1} \Lambda>0, \& b \mu_{2} \mu_{3}<q p_{1} \Lambda$, holds true

Proof: Evaluate the Jacobian matrix at $E_{3} J(S, 0, P)=$ $\left(\begin{array}{ccc}-p_{1} P-\mu_{1} & -\beta S & -p_{1} S \\ 0 & \beta S-p_{2} P-\mu_{2} & 0 \\ q p_{1} P & q p_{2} P & q p_{1} S-\mu_{3}\end{array}\right)$

To find Eigen value of the Jacobian matrix $\operatorname{Det}\left[J\left(E_{3}\right)-\lambda I\right]$

i.e. $\left|\begin{array}{ccc}-p_{1} P-\mu_{1}-\lambda & -\beta S & -p_{1} S \\ 0 & \beta S-p_{2} P-\mu_{2}-\lambda & 0 \\ q p_{1} P & q p_{2} P & q p_{1} S-\mu_{3}-\lambda\end{array}\right|$

$\left(\beta S-p_{2} P-\mu_{2}-\lambda\right)\left[\left(-p_{1} P-\mu_{1}-\lambda\right)\left(q p_{1} S-\mu_{3}-\right.\right.$

$\left.\lambda)+\mathrm{qp}_{1}{ }^{2} \mathrm{SP}\right]=0$ is stable if all Eigen values (roots) of this polynomial should be negative, otherwise unstable.

$\beta S-p_{2} P-\mu_{2}-\lambda=0$ and $\left(\lambda+p_{1} P+\mu_{1}\right)\left(\lambda+\mu_{3}-\right.$ $\left.q p_{1} S\right)+\mathrm{qp}_{1}{ }^{2} \mathrm{SP}=0$

Therefore the system is to be stable, if the Eigen value $\beta S-p_{2} P-\mu_{2}<0$, and the remaining Eigen value obtained from the roots of the quadratic equation

$\left(\lambda+p_{1} P+\mu_{1}\right)\left(\lambda+\mu_{3}-q p_{1} S\right)+\mathrm{qp}_{1}{ }^{2} \mathrm{SP}=0$ Now, Using Routh Hourwith criterion, $p_{1} P+\mu_{1}+\mu_{3}-q p_{1} S>0$ and

$\left(p_{1} P+\mu_{1}\right)\left(\mu_{3}-q p_{1} S\right)+\mathrm{qp}_{1}{ }^{2} \mathrm{SP}>0$ holds true.

Furthermore, $\beta \mu_{3}-q p_{2}\left(q p_{1} \Lambda-\mu_{1} \mu_{3}\right)<q p_{1} \mu_{2} \mu_{3}$, $q p_{1} \Lambda>0, \mu_{2} \mu_{3}<q p_{1} \Lambda$, holds true, otherwise unstable Theorem 5 [Global stability] Endemic equilibrium point $E_{4}\left(S^{*}, I^{*}, P^{*}\right)$ is globally asymptotically stable.

Proof: Let us define a liapunove function as follows 


$$
\begin{array}{r}
L(S, \quad I, \quad P)=\mathrm{m}_{1}\left(\mathrm{~s}-\mathrm{s}^{*}\right)^{2} / 2+\frac{\mathrm{m}_{2}\left(\mathrm{I}-\mathrm{I}^{*}\right)^{2}}{2} \\
+\mathrm{m}_{3}\left(\mathrm{P}-\mathrm{P}^{*}\right)^{2} / 2 \\
\begin{array}{r}
d L / d t=\mathrm{m}_{1}\left(\mathrm{~s}-\mathrm{s}^{*}\right)\{\mathrm{ds} / \mathrm{dt}\}+\mathrm{m}_{2}\left(\mathrm{I}-\mathrm{I}^{*}\right)\{\mathrm{dI} / \mathrm{dt}\}+ \\
m_{3}\left(\mathrm{P}-\mathrm{P}^{*}\right)\{d P / d t\}
\end{array}
\end{array}
$$

Now substitute the model equation (1) - (3) into (4)

$$
\begin{gathered}
\mathrm{dL} / \mathrm{dt}=\mathrm{m}_{1}\left(\mathrm{~s}-\mathrm{s}^{*}\right)\left\{\Lambda-\beta S I-p_{1} S P-\mu_{1} S\right\} \\
+\mathrm{m}_{2}\left(\mathrm{I}-\mathrm{I}^{*}\right)\left\{\beta S I-p_{2} I P-\mu_{2} I-\delta I\right\} \\
+\mathrm{m}_{3}\left(\mathrm{P}-\mathrm{P}^{*}\right)\left\{q p_{1} S P+q p_{2} I P-\mu_{3} P\right\}
\end{gathered}
$$

Take out S, I, P and put as change

$$
\begin{gathered}
\mathrm{dL} / \mathrm{dt}=\mathrm{m}_{1}\left(\mathrm{~s}-\mathrm{s}^{*}\right)\left(\mathrm{s}-\mathrm{s}^{*}\right)\left\{\left[\frac{\Lambda}{\mathrm{S}}\right]-\beta I-p_{1} P-\mu_{1}\right\} \\
+\mathrm{m}_{2}\left(\mathrm{I}-\mathrm{I}^{*}\right)\left(\mathrm{I}-\mathrm{I}^{*}\right)\left\{\beta S-p_{2} P-\mu_{2}-\delta\right\} \\
+\mathrm{m}_{3}\left(\mathrm{P}-\mathrm{P}^{*}\right)\left(\mathrm{P}-\mathrm{P}^{*}\right)\left\{q p_{1} S+q p_{2} I-\mu_{3}\right\}
\end{gathered}
$$

By rearranging and take out negative sign from the bracket it could be obtained as

$$
\begin{gathered}
\mathrm{dL} / \mathrm{dt}=-\mathrm{m}_{1}\left(\mathrm{~s}-\mathrm{s}^{*}\right)^{2}\left\{-\left[\frac{\Lambda}{\mathrm{S}}\right]+\beta I+p_{1} P+\mu_{1}\right\} \\
-\mathrm{m}_{2}\left(\mathrm{I}-\mathrm{I}^{*}\right)^{2}\left\{-\beta S+p_{2} P+\mu_{2}+\delta\right\} \\
-\mathrm{m}_{3}\left(\mathrm{~T}-\mathrm{T}^{*}\right)^{2}\left\{\left\{-q p_{1} S-q p_{2} I+\mu_{3}\right\}\right\}
\end{gathered}
$$

Thus it is possible to set $m_{1}, m_{2}, m_{3}, m_{3}$ are non negative integers such that $\mathrm{dL} / \mathrm{dt} \leq 0$ and endemic equilibrium point is globally stable.

\section{Simulation Study}

In this section, the numerical simulation of model equations (1) - (3) is carried out using the software DE Discover 2.6.4. For Simulation purpose, a set of meaningful values are assigned to the model parameters. A set of initial conditions are given to the model variables. These sets of parametric values are given in Tables 3 and Model equations and parameter is arranged for DEDiscover software in this way for simulation Study purpose.

dS/dt=lambda-beta*S*I-p_1*S*P-mu_1*S//susceptible prey

$\mathrm{dI} / \mathrm{dt}=$ beta*S*I-p_2*I*P-mu_2*I-Delta*I // Infected prey $\mathrm{dP} / \mathrm{dt}=\mathrm{q} * \mathrm{p}_{-} 1 * \mathrm{~S} * \overline{\mathrm{P}}+\mathrm{q} * \mathrm{p} \_2 * \overline{\mathrm{I}} \mathrm{P}-\mathrm{mu} \_3 * \mathrm{P} / /$ Predator

Table 3. Parameter values used for Simulation.

\begin{tabular}{lll}
\hline Parameter & Value & Reference \\
\hline$r$ & 11.2000 & {$[1]$} \\
$k$ & 30.0000 & {$[1]$} \\
$\beta$ & 1.2000 & {$[1]$} \\
$p_{1}$ & 0.4000 & {$[1]$} \\
$p_{2}$ & 0.6000 & {$[1]$} \\
$\delta$ & 0.4000 & {$[1]$} \\
$\mu_{1}$ & 0.0100 & Assumed \\
$\mu_{2}$ & 0.0800 & {$[1]$} \\
$\mu_{3}$ & 0.0200 & Assumed \\
\hline
\end{tabular}

Using the parameter values given in Table 3 and the initial conditions $S(0)=40, I(0)=25, P(0)=8$ in the model equations (1) - (3) is simulated and the results are given in Figures 2-5.

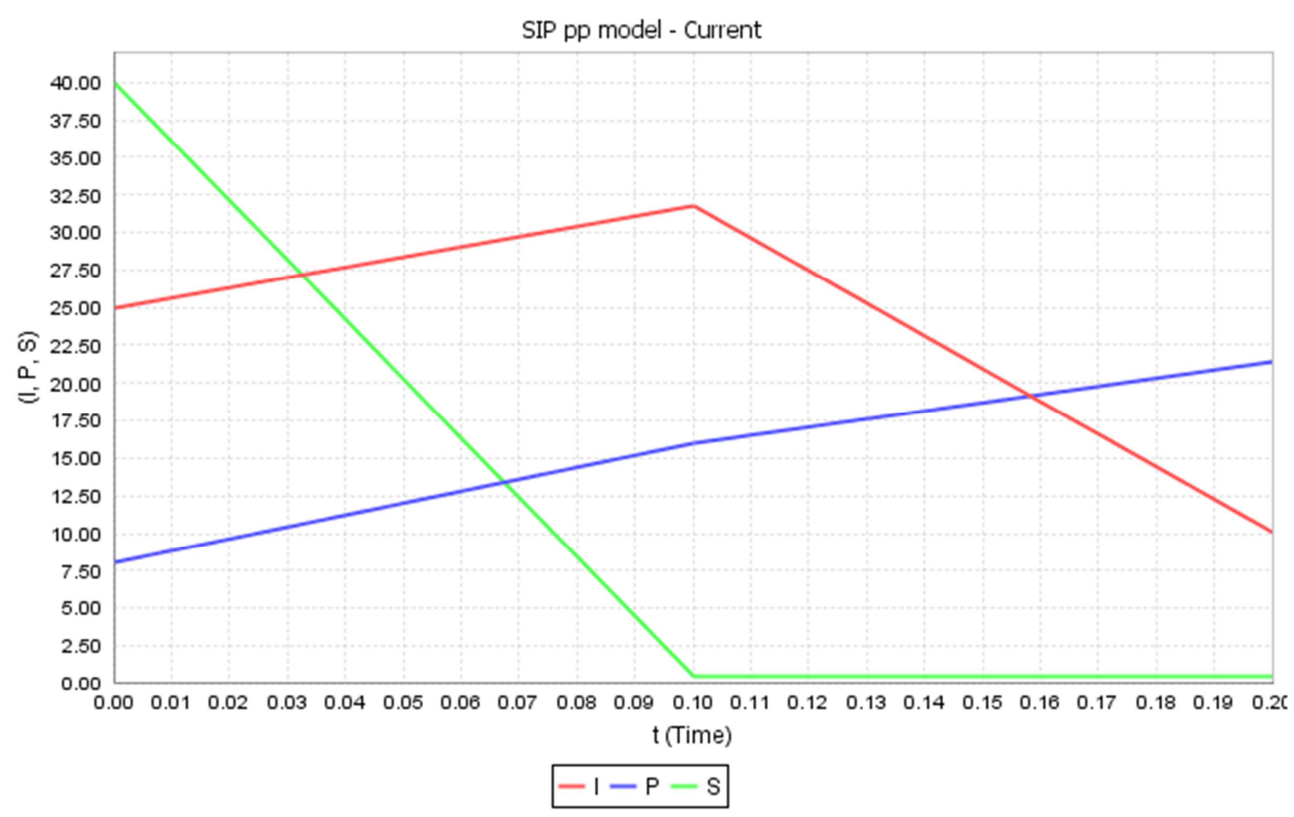

Figure 2. Time series plot for prey-predator population with $p_{1} \geq p_{2}$.

From Figure 2, it is observed that initially infected preys are increasing in number meaning more infection and predation occurs on susceptible prey that leads rapid decreasing on the number of susceptible prey, but the predator continue consuming the prey species and eventually the infected prey species were abundant in the system due to disease. This situation creates 
great chance for predator to consume infected prey. however the predators are increasing in number for some time and will decrease in the long run. Then there will be a chance for susceptible prey to grow, produce new of springs and the system will be stable.

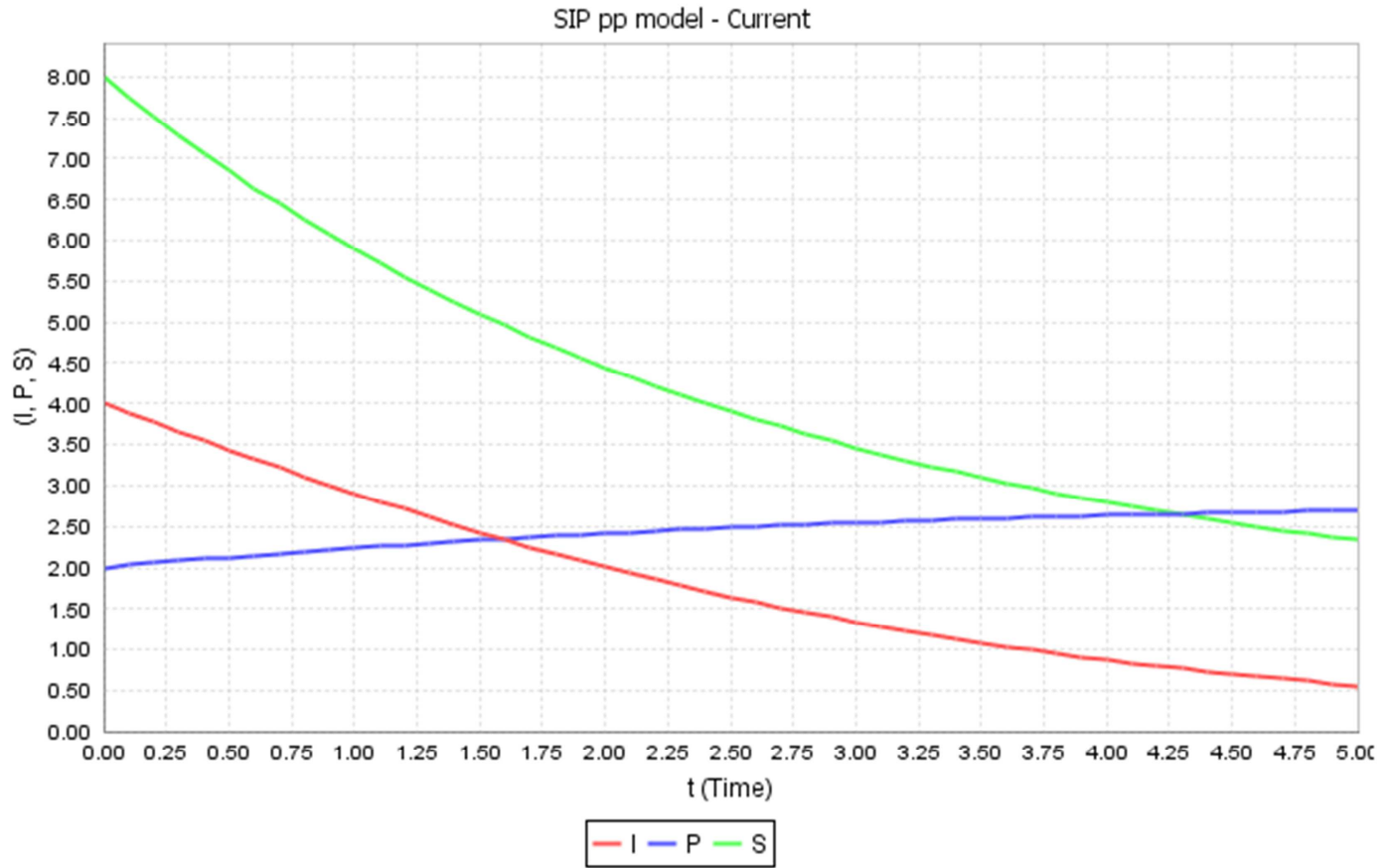

Figure 3. Time series plot for prey-predator population with $p_{1} \leq p_{2}$.

In Figure 3, Shows the number of predators increased due to different consumption rates of both infected and susceptible prey. The infected and susceptible prey are decreasing in the graph due to prey is suffering from predation and infectious disease for some time. In the long run the system may be stable due to similar reason in Figure 2.

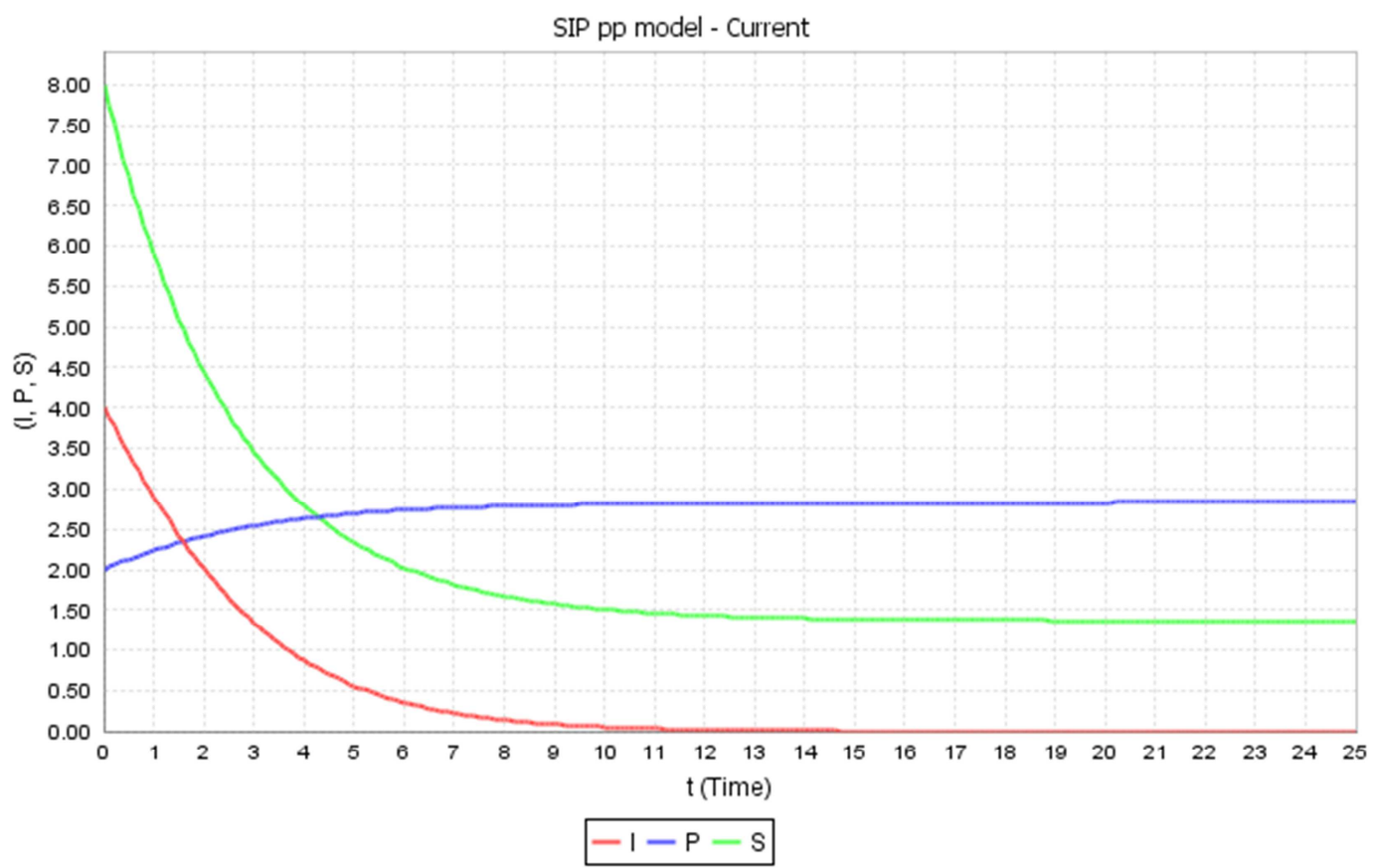

Figure 4. Time series plot for prey-predator population with different initial conditions.

Figure 4, shows that infected preys are highly exposed for predation and also they are suffering from death rate due to infection. Eventually the infected prey may be cleared out from the system and the disease die out. The Susceptible preys become healthy, grow well, and Contribute stability in the prey-predator system. The susceptible prey and predator 
graph depicts that the predator-prey system is stable after some time.

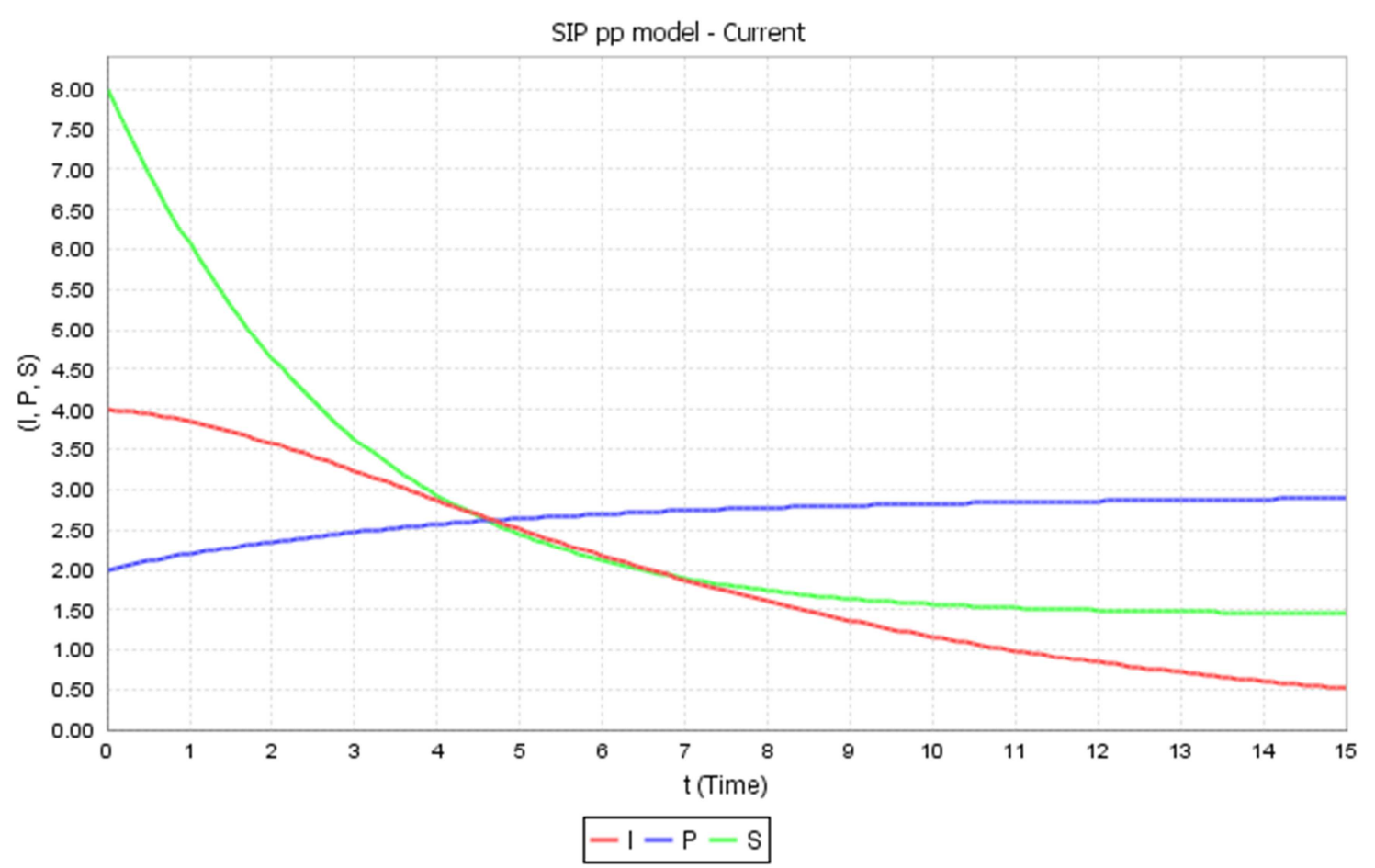

Figure 5. Time series plot for prey-predator population with different initial conditions.

Figure 5 show that the prey-predator of Susceptibleinfected prey decrease through time. eventually the population of all kind may converge to the same number Due to predators attack and infectious Disease. The total preypredator population indicate oscillations and after some time the population of prey species are minimize in number or remain stable depending on the situation of the populations.

\section{Conclusions}

Based on biologically valid and meaningful assumptions, Susceptible-infected prey and predator of eco-epidemiological model is constructed. All possible Equilibrium points are computed. Local and global stability analysis of Disease-free and endemic equilibrium points are performed.

In simulation study, it is observed that the prey species is decreasing due to infectious disease but this facilitate the opportunity of predator to consume the prey easily which turn increase in predator. On the other hand, when Infected prey completely eaten up by predators, the susceptible prey had no time to be infected by infective prey. Thus the infectious disease die out and susceptible prey would increase in number which leads the prey-predator system wait stable for some time.

\section{Acknowledgements}

The authors would like to thank the editor and the anonymous reviewers of the journal AJAM for their helpful suggestions and remarks.

\section{References}

[1] Alfred Hugo, Estomih S. Massawe, and Oluwole Daniel Makinde (2012). An Eco-Epidemiological Mathematical Model with Treatment and Disease Infection in both Prey and Predator Population. Journal of Ecology and natural environment Vol. 4 (10), pp. 266-273.

[2] Shashi Kant, Vivek Kumar, (2017) Dynamics of A PreyPredator System With Infection In Prey, Electronic Journal of Deferential Equations, Vol. 2017, No. 209, pp. 1-27.

[3] Lihong Wang, Fanghong Zhang, \& Cuncheng Jin, (2017) Analysis of an eco-epidemiological model with disease in the prey and predator International Journal of Mathematical Research Vol. 6, No. 1, pp 22-28.

[4] T. K. Kar, Prasanta Kumar Mondal, (June 20120 A Mathematical Study on the Dynamics of an EcoEpidemiological Model in the Presence of Delay, Applications and Applied Mathematics: An International Journal (AAM), Vol. 7, Issue 1, pp 300-333.

[5] Abayneh Fentie Bezabih, Geremew Kenassa Edessa, Koya Purnachandra Rao (2020), Mathematical Eco-Epidemic Model on Prey-Predator System. IOSR Journal of Mathematics (IOSR-JM), 16 (1), pp. 22-34.

[6] Rald Kamel Naji (2012) The dynamics of prey-predator model with disease in prey, research gate, Available online at http://scik.org.

[7] Xin-You Meng, Ni-Ni Qin \& Hai-Feng Huo (2018) Dynamics analysis of a predator-prey system with harvesting prey and disease in prey species, Journal of Biological Dynamics, 12: 1, 342-374, DOI: 10.1080/17513758.2018.1454515. 
[8] Rald Kamel Naji, Rasha Ali Hamodi, (August 2016) The dynamics of an ecological model with infectious disease, Global Journal of Engineering Science and Researches, DOI: 10.5281/zenodo.61221.

[9] Geremew Kenassa Edessa, Boka Kumsa, Purnachandra Rao Koya (2018). Dynamical behavior of Susceptible prey Infected prey - Predator Populations. IOSR Journal of Mathematics (IOSR-JM) 14 (4) PP: 31-41.

[10] S. P. Bera, A. Maiti, G. Samanta (2015). A Prey-predator Model with Infection in both prey and predator, Filomat 29 (8) pp, 1753-1767.

[11] Paritosh Bhattacharya, Susmita Paul and K. S. Choudhury (2015). Mathematical Modeling of Ecological Networks, Structure and Interaction Of Prey and Predator, Palestine Journal of Mathematics Vol. 4 (2), pp 335-347.

[12] Asrul Sani, Edi Cahyono, Mukhsar, Gusti Arviana Rahman (2014). Dynamics of Disease Spread in a Predator-Prey System, Indonesia, Advanced Studies in Biology, Vol. 6, No. 4, pp 169-179.
[13] C. M. Silva (2017). Existence of periodic solutions for periodic eco-epidemic models with disease in the prey, $\mathrm{J}$. Math. Anal. Appl. 453 (1), pp 383-397.

[14] M. Haque (2010). A predator-prey model with disease in the predator species only, Nonlinear Anal., Real World Appl., 11 (4), 2224-2236.

[15] M. Liu, Z. Jin and M. Haque, An impulsive predator-prey model with communicable disease in the prey species only, Nonlinear Anal. Real World Appl. 10 (2009), no. 5, 3098-3111.

[16] K. P. Hadeler and H. I. Freedman (1989). Predator-prey populations with parasitic infection, J. Math. Biol., 27, pp 609-631.

[17] M. S. Rahman and S. Chakravarty (2013). A predator-prey model with disease in prey. Nonlinear Analysis: Modeling and Control, 18 (2), pp 191-209.

[18] Sachin Kumar and Harsha Kharbanda (Sep 2017). Stability Analysis Of Prey-Predator Model With Infection, Migration and Vaccination In Prey, arXiv: 1709.10319vl [math. DS], 29. 